\title{
Vitamin D, Depressive Symptoms, and Covid-19 Pandemic
}

\author{
Gilciane Ceolin 1,2*, Giulia Pipolo Rodrigues Mano 2,3, Natália Schmitt Hames ${ }^{2,3}$, \\ Luciana da Conceição Antunes ${ }^{2,4}$, Elisa Brietzke ${ }^{5,6,7}$, Débora Kurrle Rieger ${ }^{1,2}$ and \\ Júlia Dubois Moreira ${ }^{1,2}$
}

\begin{abstract}
${ }^{1}$ Postgraduate Program in Nutrition, Federal University of Santa Catarina, Florianopolis, Brazil, ${ }^{2}$ Translational Nutritional Neuroscience Working Group, Federal University of Santa Catarina, Florianopolis, Brazil, ${ }^{3}$ Nutrition Graduation Course, Federal University of Santa Catarina, Florianopolis, Brazil, ${ }^{4}$ Department of Nutrition, Federal University of Santa Catarina, Florianopolis, Brazil, ${ }^{5}$ Department of Psychiatry, Queen's University, Kingston, ON, Canada, ${ }^{6}$ Inpatient Psychiatric Unit, Kingston General Hospital, Kingston Health Sciences Centre, Kingston, ON, Canada, ${ }^{7}$ Centre for Neuroscience Studies, Queen's University, Kingston, ON, Canada
\end{abstract}

Since the COVID-19 outbreak, studies across diverse countries have strongly pointed toward the emergence of a mental health crisis, with a dramatic increase in the prevalence of depressive psychopathology and suicidal tendencies. Vitamin D deficiency has been associated with an increased risk of mental health problems as well as individual responses to stress. Studies have discussed the relationship between low

OPEN ACCESS

Edited by:

Vittorio Calabrese,

University of Catania, Italy

Reviewed by:

Keiko Iwata,

University of Fukui, Japan

Reza Rastmanesh,

Independent researcher, Tehran, Iran

*Correspondence:

Gilciane Ceolin

nutrigil@live.com

Specialty section:

This article was submitted to Neuroenergetics, Nutrition and Brain

Health,

a section of the journal

Frontiers in Neuroscience

Received: 22 February 2021

Accepted: 12 April 2021

Published: 13 May 2021

Citation:

Ceolin G, Mano GPR, Hames NS, Antunes LC, Brietzke E, Rieger DK and Moreira JD (2021) Vitamin D, Depressive Symptoms, and Covid-19

Pandemic.

Front. Neurosci. 15:670879. doi: 10.3389/fnins.2021.670879 serum vitamin D concentrations and depressive symptoms, suggesting that maintaining adequate concentrations of serum vitamin $D$ seems to have a protective effect against it. Vitamin D was found to contribute to improved serotonergic neurotransmission in the experimental model of depression by regulating serotonin metabolism. The signaling of 1,25-dihydroxyvitamin $D 3$, the active form of vitamin $D$, through vitamin $D$ receptor (VDR) induces the expression of the gene of tryptophan hydroxylase 2 (TPH2), influences the expression of serotonin reuptake transporter (SERT) as well as the levels of monoamine oxidase-A (MAO-A), the enzyme responsible for serotonin catabolism. Vitamin $D$ also presents a relevant link with chronobiological interplay, which could influence the development of depressive symptoms when unbalance between light-dark cycles occurs. In this Perspective, we discussed the significant role of vitamin D in the elevation of stress-related depressive symptoms during the COVID-19 pandemic. It is suggested that vitamin D monitoring and, when deficiency is detected, supplementation could be considered as an important healthcare measure while lockdown and social isolation procedures last during the COVID-19 pandemic.

Keywords: vitamin D, cholecalciferol, depressive symptoms, social isolation, pandemics, CoVID-19

\section{DEPRESSION AND COVID-19 PANDEMIC}

The current pandemic of coronavirus (COVID-19) has imposed social isolation measures, forcing many people to stay confined at home, canceling routine outdoor activities (working routine, sidewalks, exercises in fitness centers, and park visits), and limiting social interaction among friends, coworkers, and family. A study concentrated on a general population from China within the first 2 weeks of the COVID-19 outbreak indicated that the lifestyle 


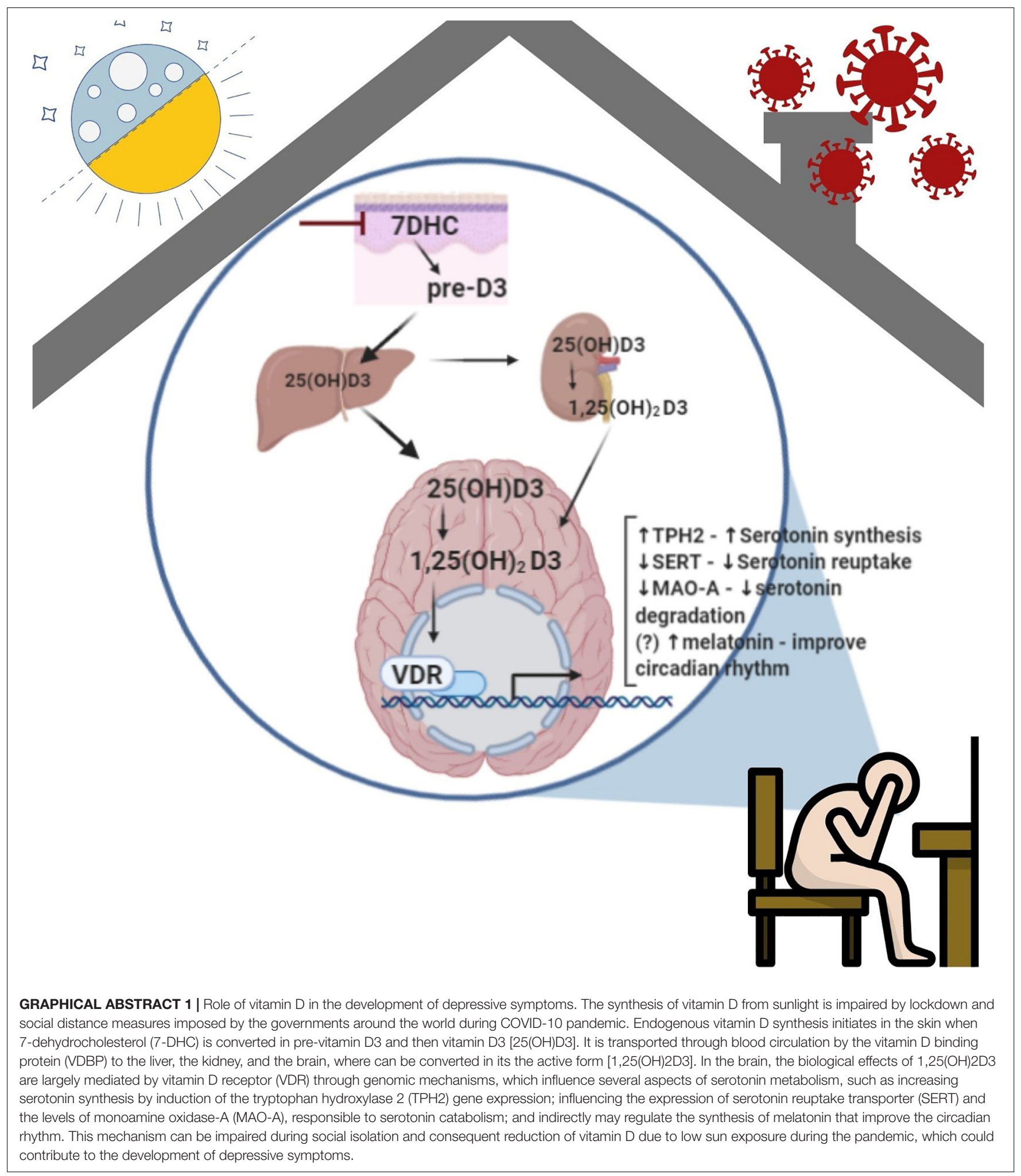

changes prompted individuals to stay indoors for over $20-24 \mathrm{~h}$ per day (Wang et al., 2020).

Since the COVID-19 outbreak, studies across diverse countries have strongly pointed toward the emergence of a mental health crisis, with a dramatic increase in the prevalence of depressive psychopathology and suicidal tendencies. A systematic review of the instances of mental health conditions during the period of the COVID-19 pandemic showed that the prevalence 
of depressive symptoms ranged from 14.6 to $48.3 \%$ across all populations (China, Spain, Italy, Iran, the United States, Turkey, Nepal, and Denmark) (Xiong et al., 2020). In the United States, the prevalence of symptoms associated with depression increased more than threefold during the COVID-19 pandemic compared to the times before the viral outbreak (8.5\% before to $27.8 \%$ during COVID-19) (Ettman et al., 2020). In London, after lockdown the prevalence of feeling worse on the depression was $12.8 \%$ and to anxiety was $12.3 \%$ in people with 50 years or over (Robb et al., 2020). Restriction of public circulation and social isolation, despite those being essential measures to contain the pandemic, has been postulated to be responsible for increased stress, loneliness, and unhealthy lifestyle habits (Luo et al., 2020; Smith and Lim, 2020).

Recently, a longitudinal study involving older adults (Santini et al., 2020) demonstrated that social disconnectedness could contribute to a greater risk of depression and anxiety, which has also been reported in adolescent and adult populations (Smith and Lim, 2020). In a survey conducted by UNICEF, Australia on 1,000 young people (aged 13-17 years), almost half of the respondents asserted that COVID-19 had negatively affected their levels of stress and anxiety. An elevated level of stress, fear, irritability, frustration, and boredom was reported by individuals who were in social isolation and had experienced a major deflection from their usual routine and interaction with other people (Smith and Lim, 2020). In addition to the increase in the risk of mental health problems, the pandemic has also exacerbated preexisting psychiatric conditions. Vulnerability to psychological stress might depend on social support, isolation time, COVID-19 infection-related factors, the amount of mass media exposure, physical activity, and eating patterns (Kontoangelos et al., 2020).

\section{VITAMIN D AND SUNLIGHT EXPOSURE: BREAKDOWN BY SOCIAL ISOLATION MEASURES DURING COVID-19 PANDEMIC}

Vitamin D deficiency has been associated with an increased risk of mental health problems as well as individual responses to stress (Amrein et al., 2020). There are several risk factors related to vitamin D deficiency such as obesity, dark skin, living in countries with low sunlight incidence, gastrointestinal malabsorption, renal insufficiency, liver disease, and the use of covered clothing and sunscreen (Kennel et al., 2010; Holick et al., 2011). Furthermore, reduced exposure to sunlight, thereby reducing the biosynthesis of vitamin D in the skin, is a strong factor in the pathophysiology of vitamin D deficiency and studies have been demonstrated that sun exposure can enhance vitamin D synthesis (Jorde et al., 2010; Chel et al., 2011; Macdonald et al., 2011; Chalcraft et al., 2020).

After adequate exposure to sunlight, the conversion of 7-dehydrocholesterol (7-DHC) to 25-hydroxycholecalciferol $[25(\mathrm{OH}) \mathrm{D}]$ takes approximately $8 \mathrm{~h}$ and an additional time to enter the dermal capillary bed. The daily exposure of $20 \%$ of the body surface is sufficient to increase $25(\mathrm{OH}) \mathrm{D}$, which points toward the importance of sunlight in the maintenance of vitamin D levels at the appropriate concentration (Wacker and Holick, 2013). The half-life of $25(\mathrm{OH}) \mathrm{D}$ was estimated to 15 to 25 days, approximately (Lips, 2007; Jones et al., 2014). The half-life of serum $25(\mathrm{OH}) \mathrm{D}$ was estimated to be $\sim 82$ days when calculated after one single loading dose of 100000 IU of cholecalciferol was administered at the beginning of the study, followed by a daily dose of 4800 IU from days 7 to 20, and none from day 21 (Oliveri et al., 2015). In Nordic countries, which experience low amounts of sunlight, vitamin D concentrations were found to be reduced by 20 to $40 \%$ during the winter (Jorde et al., 2010). These data suggest that vitamin $\mathrm{D}$ supplementation is crucial in individuals who experience long periods of indoor activities and/or low sunshine exposure.

Social isolation and lockdown measures caused a reduction in the time spent outdoors and possibly less exposure to sunlight necessary to maintain vitamin $\mathrm{D}$ concentrations. When associated with a change in eating habits, with a predominance of meals ordered through food delivery joints, which have a reduced nutritional and vitamin $\mathrm{D}$ content, it could also reduce the daily amount of vitamin $\mathrm{D}$ for organism maintenance. One study conducted in Verona (Italy) during the COVID-19 pandemic, which intends to investigate whether social isolation could impact the vitamin D status of the general population, conclude that this was not an issue in Verona (the region in which data was collected) (Lippi et al., 2021). Despite scarce publications address this issue, research groups, and professionals already start to concern about vitamin $\mathrm{D}$ deficiency during the COVID-19 pandemic. Some postulate that home reclusion could lead to a surge of vitamin D deficiency around the world, which has been associated with developing type 2 diabetes, cognitive decline, malignant neoplasms, autoimmune diseases, cardiovascular diseases, osteoporosis, risk of fall in the elderly, and overall mortality (Alpalhão and Filipe, 2020). In fact, the diminished vitamin $\mathrm{D}$ intake and sun exposure might result in severe manifestations since the worldwide prevalence of vitamin $\mathrm{D}$ deficiency had been documented as a health concern even before the pandemic (Luo et al., 2018; Zhou et al., 2019; Amrein et al., 2020).

\section{VITAMIN D AND DEPRESSIVE SYMPTOMS}

Vitamin D low levels have been previously associated with the risk of depressive symptoms and depression worldwide (Song et al., 2016; Vidgren et al., 2018; Yao et al., 2018; Ceolin et al., 2020; Köhnke et al., 2020). However, few publications have been discussing this relationship during the COVID-19 pandemic (Di Nicola et al., 2020; Mehta et al., 2021). Mehta et al. (2021) discussed that vitamin D can be hypothesized to trigger as well as sustain the psychiatric symptoms and can also be expected to alleviate the psychiatric manifestations in COVID-19. An observational study in Rome showed that low 25hydroxyvitamin D serum levels were significantly associated with higher psychological distress in patients with mood disorders during the COVID-19 outbreak (Di Nicola et al., 2020). 
The mechanisms associated with vitamin $\mathrm{D}$ antidepressant effects at the cellular level are under investigation in experimental studies, and some hypotheses have been presented in different reports in the literature. The VDR and some cytochrome P450 enzymes, which are responsible for the transformation of vitamin $\mathrm{D}$ into its active form, were found in cerebral cells and in brain areas that have been implicated in the pathophysiology of depression (He et al., 2020). The optimal vitamin D status was found to contribute to improved serotonergic neurotransmission deficits in an experimental model of depression by regulating serotonin synthesis (Sabir et al., 2018). To increase serotonin concentrations, tryptophan must first be transported across the blood-brain barrier and metabolized by TPH2. The signaling of 1,25-dihydroxycholecalciferol [1,25(OH)2D3], the active form of vitamin $\mathrm{D}$, through VDR induces the expression of the gene TPH2 (Kaneko et al., 2015; Patrick and Ames, 2015). Moreover, $1,25(\mathrm{OH}) 2 \mathrm{D} 3$ influences the expression of SERT as well as the levels of MAO-A, the enzyme responsible for serotonin catabolism (Sabir et al., 2018).

Another important aspect related to vitamin $\mathrm{D}$ and brain function is its link with chronobiological interplay. Indeed, the interface between vitamin $\mathrm{D}$ and the circadian system has been revealed as both plasma concentrations of $1,25(\mathrm{OH}) 2 \mathrm{D} 3$ and vitamin D binding protein (DBP) display circadian oscillation patterns (Jones et al., 2017). However, available evidence suggests a mediatory role of vitamin $\mathrm{D}$ in the sleep-wake cycle, since the lower concentrations of vitamin D were correlated with impaired sleep quality and abbreviated sleep duration (Jones et al., 2017; Muscogiuri et al., 2019). Melatonin, a hormone involved in the regulation of circadian rhythm, is synthesized from the metabolism of serotonin. The widespread presence of VDR has been documented in postmortem human brain samples, in areas involved in sleep regulation, which are also brain regions that showed the strongest expression of the enzyme 1-alpha-hydroxylase, enzymes responsible for the formation of $1,25(\mathrm{OH}) 2 \mathrm{D} 3$ (Eyles et al., 2005). In this way, it is possible to hypothesize that the chronic combined deficits of vitamin $\mathrm{D}$ and sleep-wake cycle impairment due to social isolation measures and lockdown might play an essential role as a modulator of amplified depressive symptomatology and/or the pathophysiology of major depressive disorder (MDD).

Depression has also been associated with mitochondrial dysfunctions and an increase in the formation of reactive oxygen species (ROS) inducing redox to unbalance and reduction in the energy production (adenosine triphosphateATP) (Caruso et al., 2019). Oxidative stress activates several transcription factors, such as nuclear factor kappa B (NF-kB) leading to the production of pro-inflammatory cytokines and other inflammatory molecules acting as potent inducers of inducible nitric oxide synthase (iNOS) (Morris and Berk, 2015). Inflammation may induce depression through many mechanisms such as an alteration in the formation of key transmitters such as serotonin and an increase in $\mathrm{Ca}^{2+}$ signaling (Leonard and Maes, 2012; Berridge, 2017). Also, dysfunction in mechanistic target of rapamycin (mTOR) signaling can lead to ROS production and the mTOR inhibition enhance sirtuin signaling contributing to mitochondrial integrity (Mocayar Marón et al., 2020). Vitamin D and melatonin share common signaling pathways that mediate homeostatic mitochondrial function, which includes the downregulation of mTOR, iNOS, and NF- $\mathrm{BB}$ pathways that are involved in increasing oxidative stress and cell damage. Also, Vitamin $\mathrm{D}$ and melatonin decrease oxidative stress by upregulation of sirtuin 1 (SIRT-1) and adenosine monophosphateactivated protein kinase (AMPK) pathways and activating antioxidant defense pathways by expression of antioxidant proteins. These pathways are crucial to avoid abnormal inflammatory responses related to oxidative stress and apoptosis (Mocayar Marón et al., 2020).

The studies aiming to investigate the antidepressant effect of vitamin D supplementation when depression was already diagnosed observed conflicting results (Alavi et al., 2019; Alghamdi et al., 2020; Okereke et al., 2020; Vellekkatt et al., 2020; Zhu et al., 2020). To mention some of them, in randomized clinical trials (RCT), Vellekkatt et al. (2020), Alghamdi et al. (2020), and Alavi et al. (2019) observed a reduction of depressive symptoms after vitamin D supplementation in patients with different stages of depression. Nevertheless, Okereke et al. (2020) and Zhu et al. (2020) did not observe improvements in depressive symptoms after vitamin D supplementation. Data from a previous meta-analysis with RCT showed that supplementation with vitamin $\mathrm{D}(\geq 800$ I.U. daily dose) had a positive effect when compared to the effect of antidepressants (Spedding, 2014). But, a separate meta-analysis did not support this evidence in adults and pointed out that the variability in methodological attributes of the studies included in the meta-analysis (differences in vitamin D doses, time of intervention duration, and assessment of depression) could influence the conclusions (Gowda et al., 2015). Despite all this uncertainty about the effects of vitamin $\mathrm{D}$ supplementation to treat depression, when the preventive effect of adequate concentrations of vitamin D was investigated in observational studies, the results are promising. Two systematic reviews and meta-analysis with population-based epidemiological studies (Ju et al., 2013; Li et al., 2019) showed an inverse association between serum $25(\mathrm{OH}) \mathrm{D}$ and the risk of depression and, in the pooled estimation analysis, an increase of $10 \mathrm{ng} / \mathrm{mL}$ in serum concentrations of vitamin $\mathrm{D}$ might have a protective effect against depression. The data from these two metaanalyses leads us to postulate that the monitoring of vitamin $\mathrm{D}$ during long periods of indoor activities associated with reduced time to sunlight exposure is a reasonable health measure, as well as the supplementation to maintain adequate vitamin D concentrations.

\section{VITAMIN D AND COVID-19}

A growing body of literature has been discussing a possible relationship between 25(OH)D and COVID-19, in which vitamin $\mathrm{D}$ may contribute to reducing the severity of illness caused by COVID-19 (Bergman, 2020; Jakovac, 2020; Marik et al., 2020; Mitchell, 2020; Panarese and Shahini, 2020). The main discussion is that Vitamin D could exert protective effects against respiratory infections, due to its immunomodulatory and anti-inflammatory roles, which have been highlighted 
in patients with community-acquired infections and acute respiratory failure (Zhou et al., 2019; Bergman, 2020; Mitchell, 2020; Panarese and Shahini, 2020). COVID-19 has been shown to affect the neuroendocrine-immune system wherein it suppresses its activity. The neuroendocrine-immune system has been implicated in stress response and coping strategies (Nami et al., 2020).

One study performed in vitro showed that the active form of Vitamin D, calcitriol, exhibits significant potent activity against SARS-CoV-2 in African green monkey kidney cells, human hepatoma cells, and human nasal epithelial cells (Mok et al., 2020). In fact, the interest in the field has been growing and some systematic reviews and meta-analysis were performed to measure the risk of infection and severity of COVID-19 due to low levels of 25(OH)D in observational studies (Pereira et al., 2020; Kazemi et al., 2021; Liu et al., 2021; Petrelli et al., 2021; Teshome et al., 2021). Pereira et al. (2020) reported that severe cases of COVID-19 present $64 \%$ (OR $=1.64$; 95\% CI $=1.30$ 2.09) of vitamin $\mathrm{D}$ deficiency and that insufficiency increased hospitalization $(\mathrm{OR}=1.81 ; 95 \% \mathrm{CI}=1.41-2.21)$ and mortality (OR $=1.82 ; 95 \%$ CI $=1.06-2.58)$. Kazemi et al. (2021) found that vitamin $\mathrm{D}$ deficiency was a higher risk of SARS-CoV-2 infection $(\mathrm{OR}=1.77 ; 95 \% \mathrm{CI}=1.24-2.53)$ and presented a higher severity $(\mathrm{OR}=2.57 ; 95 \% \mathrm{CI}=1.65-4.01)$. Liu et al. (2021) showed that vitamin D deficiency or insufficiency also was associated with an increased risk of COVID-19 (OR = 1.43; 95\% CI = 1.00-2.05). Petrelli et al. (2021) revealed that deficient vitamin $\mathrm{D}$ values showed a higher risk of COVID-19 infection $(\mathrm{OR}=1.26 ; 95 \% \mathrm{CI}=1.19-1.34)$ and worse severity $(\mathrm{OR}=2.6$; $95 \% \mathrm{CI}=1.84-3.67)$ and higher mortality $(\mathrm{OR}=1.22 ; 95 \% \mathrm{CI}$, 1.04-1.43). Teshome et al. (2021) found that vitamin D deficiency was $80 \%$ more likely to acquire COVID-19 infection $(\mathrm{OR}=1.80$; $95 \% \mathrm{CI}=1.72-1.88)$.

Concerning clinical trials, only one systematic review and metanalysis were performed to identify the effect of vitamin D on COVID-19, and 32 clinical protocol was identified but only three studies have published results to-date and the results are controversial (Bassatne et al., 2021). In fact, there are some challenges in vitamin $\mathrm{D}$ research and some authors have provided recommendations for the design of randomized clinical trials of

\section{REFERENCES}

Alavi, N. M., Khademalhoseini, S., Vakili, Z., and Assarian, F. (2019). Effect of vitamin $\mathrm{D}$ supplementation on depression in elderly patients: a randomized clinical trial. Clin. Nutr. 38, 2065-2070. doi: 10.1016/j.clnu.2018.09.011

Alghamdi, S., Alsulami, N., Khoja, S., Alsufiani, H., Tayeb, H. O., and Tarazi, F. I. (2020). Vitamin D supplementation ameliorates severity of major depressive disorder. J. Mol. Neurosci. 70, 230-235. doi: 10.1007/s12031-019-01461-2

Alpalhão, M., and Filipe, P. (2020). SARS-CoV-2 pandemic and vitamin D deficiency-a double trouble. Photodermatol. Photoimmunol. Photomed. 36, 412-413. doi: 10.1111/phpp.12579

Amrein, K., Scherkl, M., Hoffmann, M., Neuwersch-Sommeregger, S., Köstenberger, M., Tmava Berisha, A., et al. (2020). Vitamin D deficiency 2.0: an update on the current status worldwide. Eur. J. Clin. Nutr. 74, 1498-1513. doi: 10.1038/s41430-020-0 558-y

Bassatne, A., Basbous, M., Chakhtoura, M., Zein, O. E., Rahme, M., and Fuleihan, G. E.-H. (2021). The link between COVID-19 and VItamin D (VIVID): vitamin D supplementation to prevent COVID-19 and to provide evidence-based guidance for clinicians and public health leaders (Camargo and Martineau, 2020).

\section{CONCLUSION}

In the light of these evidence, it is possible to postulate that vitamin $\mathrm{D}$ deficiency could play a significant role in the elevation of stress-related depressive symptoms during the COVID-19 pandemic. It has been postulated that vitamin $\mathrm{D}$ is involved in the serotoninergic system, influencing serotonin metabolism and contributing to circadian rhythm maintenance, which are important aspects of the development of depressive symptoms. In order to reduce physical and mental health risks associated with vitamin $\mathrm{D}$ deficiency, it is suggested that vitamin $\mathrm{D}$ monitoring and, when deficiency is detected, supplementation could be considered as an important healthcare measure while lockdown and restrictive social isolation procedures last during the COVID-19 pandemic.

\section{DATA AVAILABILITY STATEMENT}

The original contributions presented in the study are included in the article/supplementary material, further inquiries can be directed to the corresponding author.

\section{AUTHOR CONTRIBUTIONS}

All authors listed have made a substantial, direct and intellectual contribution to the work, and approved it for publication.

\section{FUNDING}

GC receive Ph.D. scholarship from Coordenação de Aperfeiçoamento de Pessoal de Nível Superior-CAPES/Brazil (Finance Code 001).

a systematic review and meta-analysis. Metabolism 154753. doi: 10.1016/j. metabol.2021.154753

Bergman, P. (2020). The link between vitamin D and COVID-19: distinguishing facts from fiction. J. Intern. Med. 289, 131-133. doi: 10.1111/joim.13158

Berridge, M. J. (2017). Vitamin D and depression: cellular and regulatory mechanisms. Pharmacol. Rev. 69, 80-92. doi: 10.1124/pr.116.013227

Camargo, C. A., and Martineau, A. R. (2020). Vitamin D to prevent COVID19: recommendations for the design of clinical trials. FEBS J. 287, 3689-3692. doi: $10.1111 /$ febs.15534

Caruso, G., Benatti, C., Blom, J. M. C., Caraci, F., and Tascedda, F. (2019). The many faces of mitochondrial dysfunction in depression: from pathology to treatment. Front. Pharmacol. 10:995. doi: 10.3389/fphar.2019.00995

Ceolin, G., Matsuo, L. H., Confortin, S. C., D’Orsi, E., Rieger, D. K., and Moreira, J. D. (2020). Lower serum 25-hydroxycholecalciferol is associated with depressive symptoms in older adults in Southern Brazil. Nutr. J. 19:123. doi: 10.1186/s12937-020-00638-5

Chalcraft, J. R., Cardinal, L. M., Wechsler, P. J., Hollis, B. W., Gerow, K. G., Alexander, B. M., et al. (2020). Vitamin D synthesis following a single bout 
of sun exposure in older and younger men and women. Nutrients 12:2237. doi: 10.3390/nu12082237

Chel, V. G. M., Ooms, M. E., Pavel, S., de Gruijl, F., Brand, A., and Lips, P. (2011). Prevention and treatment of vitamin D deficiency in Dutch psychogeriatric nursing home residents by weekly half-body UVB exposure after showering: a pilot study. Age Ageing 40, 211-214. doi: 10.1093/ageing/afq159

Di Nicola, M., Dattoli, L., Moccia, L., Pepe, M., Janiri, D., Fiorillo, A., et al. (2020). Serum 25-hydroxyvitamin D levels and psychological distress symptoms in patients with affective disorders during the COVID-19 pandemic. Psychoneuroendocrinology 122:104869. doi: 10.1016/j.psyneuen.2020.104869

Ettman, C. K., Abdalla, S. M., Cohen, G. H., Sampson, L., Vivier, P. M., and Galea, S. (2020). Prevalence of depression symptoms in US adults before and during the COVID-19 pandemic. JAMA Netw. Open 3:e2019686. doi: 10.1001/ jamanetworkopen.2020.19686

Eyles, D. W., Smith, S., Kinobe, R., Hewison, M., and McGrath, J. J. (2005). Distribution of the Vitamin D receptor and $1 \alpha$-hydroxylase in human brain. J. Chem. Neuroanat. 29, 21-30. doi: 10.1016/j.jchemneu.2004.08.006

Gowda, U., Mutowo, M. P., Smith, B. J., Wluka, A. E., and Renzaho, A. M. N. (2015). Vitamin D supplementation to reduce depression in adults: metaanalysis of randomized controlled trials. Nutrition 31, 421-429. doi: 10.1016/ j.nut.2014.06.017

He, Y., Wu, Z., Lan, T., Wang, Y., Tian, Y., Chen, X., et al. (2020). The $25(\mathrm{OH}) \mathrm{D} / \mathrm{VDR}$ signaling may play a role in major depression. Biochem. Biophys. Res. Commun. 523, 405-410. doi: 10.1016/j.bbrc.2019.12.071

Holick, M. F., Binkley, N. C., Bischoff-Ferrari, H. A., Gordon, C. M., Hanley, D. A., Heaney, R. P., et al. (2011). Evaluation, treatment, and prevention of vitamin D deficiency: an endocrine society clinical practice guideline. J. Clin. Endocrinol. Metab. 96, 1911-1930. doi: 10.1210/jc.2011-0385

Jakovac, H. (2020). COVID-19 and vitamin D-is there a link and an opportunity for intervention? Am. J. Physiol. Endocrinol. Metab. 318:E589. doi: 10.1152/ ajpendo.00138.2020

Jones, K. S., Assar, S., Harnpanich, D., Bouillon, R., Lambrechts, D., Prentice, A., et al. (2014). 25(OH)D2 half-life is shorter than 25(OH)D3 half-life and is influenced by DBP concentration and genotype. J. Clin. Endocrinol. Metab. 99, 3373-3381. doi: 10.1210/jc.2014-1714

Jones, K. S., Redmond, J., Fulford, A. J., Jarjou, L., Zhou, B., Prentice, A., et al. (2017). Diurnal rhythms of vitamin D binding protein and total and free vitamin D metabolites. J. Steroid Biochem. Mol. Biol. 172, 130-135. doi: 10.1016/ j.jsbmb.2017.07.015

Jorde, R., Sneve, M., Hutchinson, M., Emaus, N., Figenschau, Y., and Grimnes, G. (2010). Tracking of serum 25-hydroxyvitamin D levels during 14 years in a population-based study and during 12 months in an intervention study. Am. J. Epidemiol. 171, 903-908. doi: 10.1093/aje/kwq005

Ju, S.-Y., Lee, Y.-J., and Jeong, S.-N. (2013). Serum 25-hydroxyvitamin D levels and the risk of depression: a systematic review and meta-analysis. J. Nutr. Health Aging 17, 447-455. doi: 10.1007/s12603-012-0418-0

Kaneko, I., Sabir, M. S., Dussik, C. M., Whitfield, G. K., Karrys, A., Hsieh, J.-C., et al. (2015). 1,25-Dihydroxyvitamin D regulates expression of the tryptophan hydroxylase 2 and leptin genes: implication for behavioral influences of vitamin D. FASEB J. 29, 4023-4035. doi: 10.1096/fj.14-269811

Kazemi, A., Mohammadi, V., Aghababaee, S. K., Golzarand, M., Clark, C. C. T., and Babajafari, S. (2021). Association of vitamin D status with SARS-CoV-2 infection or COVID-19 severity: a systematic review and meta-analysis. $A d v$. Nutr. doi: 10.1093/advances/nmab012

Kennel, K. A., Drake, M. T., and Hurley, D. L. (2010). Vitamin D deficiency in adults: when to test and how to treat. Mayo Clin. Proc. 85, 752-757; quiz 757-758. doi: 10.4065/mcp.2010.0138

Köhnke, C., Herrmann, M., and Berger, K. (2020). Associations of major depressive disorder and related clinical characteristics with 25-hydroxyvitamin D levels in middle-aged adults. Nutr. Neurosci. 1-10. doi: 10.1080/1028415X.2020. 1843892

Kontoangelos, K., Economou, M., and Papageorgiou, C. (2020). Mental health effects of COVID-19 pandemia: a review of clinical and psychological traits. Psychiatry Investig. 17, 491-505. doi: 10.30773/pi.2020.0161

Leonard, B., and Maes, M. (2012). Mechanistic explanations how cell-mediated immune activation, inflammation and oxidative and nitrosative stress pathways and their sequels and concomitants play a role in the pathophysiology of unipolar depression. Neurosci. Biobehav. Rev. 36, 764-785. doi: 10.1016/j. neubiorev.2011.12.005

Li, H., Sun, D., Wang, A., Pan, H., Feng, W., Ng, C. H., et al. (2019). Serum 25-hydroxyvitamin D levels and depression in older adults: a dose-response meta-analysis of prospective cohort studies. Am. J. Geriatr. Psychiatry 27, 1192-1202. doi: 10.1016/j.jagp.2019.05.022

Lippi, G., Ferrari, A., and Targher, G. (2021). Is COVID-19 lockdown associated with vitamin D deficiency? Eur. J. Public Health doi: 10.1093/eurpub/ckab004

Lips, P. (2007). Relative value of $25(\mathrm{OH}) \mathrm{D}$ and $1,25(\mathrm{OH}) 2 \mathrm{D}$ measurements. J. Bone Miner. Res. 22, 1668-1671. doi: 10.1359/jbmr.070716

Liu, N., Sun, J., Wang, X., Zhang, T., Zhao, M., and Li, H. (2021). Low vitamin D status is associated with coronavirus disease 2019 outcomes: a systematic review and meta-analysis. Int. J. Infect. Dis. 104, 58-64. doi: 10.1016/j.ijid.2020.12.077

Luo, J., Quan, Z., Lin, S., and Cui, L. (2018). The association between blood concentration of 25- hydroxyvitamin D and sarcopenia: a meta-analysis. Asia Pac. J. Clin. Nutr. 27, 1258-1270. doi: 10.6133/apjcn.201811_27(6).0013

Luo, M., Guo, L., Yu, M., Jiang, W., and Wang, H. (2020). The psychological and mental impact of coronavirus disease 2019 (COVID-19) on medical staff and general public - a systematic review and meta-analysis. Psychiatry Res. 291:113190. doi: 10.1016/j.psychres.2020.113190

Macdonald, H. M., Mavroeidi, A., Fraser, W. D., Darling, A. L., Black, A. J., Aucott, L., et al. (2011). Sunlight and dietary contributions to the seasonal vitamin D status of cohorts of healthy postmenopausal women living at northerly latitudes: a major cause for concern? Osteoporos. Int. 22, 2461-2472. doi: 10. 1007/s00198-010-1467-z

Marik, P. E., Kory, P., and Varon, J. (2020). Does vitamin D status impact mortality from SARS-CoV-2 infection? Med. Drug Discov. 6:100041. doi: 10. 1016/j.medidd.2020.100041

Mehta, A., Soni, V. K., Sharma, K., Ratre, Y. K., Shukla, D., Singh, A. K., et al. (2021). Finding Horcrux of psychiatric symptoms in COVID-19: deficiencies of amino acids and vitamin D. Asian J. Psychiatr. 55:102523. doi: 10.1016/j.ajp. 2020.102523

Mitchell, F. (2020). Vitamin-D and COVID-19: do deficient risk a poorer outcome? Lancet Diabetes Endocrinol. 8:570. doi: 10.1016/S2213-8587(20)30183-2

Mocayar Marón, F. J., Ferder, L., Reiter, R. J., and Manucha, W. (2020). Daily and seasonal mitochondrial protection: unraveling common possible mechanisms involving vitamin D and melatonin. J. Steroid Biochem. Mol. Biol. 199:105595. doi: 10.1016/j.jsbmb.2020.105595

Mok, C. K., Ng, Y. L., Ahidjo, B. A., Lee, R. C. H., Loe, M. W. C., Liu, J., et al. (2020). Calcitriol, the active form of vitamin D, is a promising candidate for COVID-19 prophylaxis. bioRxiv [Preprint] doi: 10.1101/2020.06.21.162396

Morris, G., and Berk, M. (2015). The many roads to mitochondrial dysfunction in neuroimmune and neuropsychiatric disorders. BMC Med. 13:68. doi: 10.1186/ s12916-015-0310-y

Muscogiuri, G., Barrea, L., Scannapieco, M., Di Somma, C., Scacchi, M., Aimaretti, G., et al. (2019). The lullaby of the sun: the role of vitamin D in sleep disturbance. Sleep Med. 54, 262-265. doi: 10.1016/j.sleep.2018.10.033

Nami, M., Gadad, B. S., Chong, L., Ghumman, U., Misra, A., Gadad, S. S., et al. (2020). The interrelation of neurological and psychological symptoms of COVID-19: risks and remedies. J. Clin. Med. 9:2624. doi: 10.3390/jcm9082624

Okereke, O. I., Reynolds, C. F., Mischoulon, D., Chang, G., Vyas, C. M., Cook, N. R., et al. (2020). Effect of long-term vitamin D3 supplementation vs placebo on risk of depression or clinically relevant depressive symptoms and on change in mood scores: a randomized clinical trial. JAMA 324, 471-480. doi: 10.1001/jama.2020. 10224

Oliveri, B., Mastaglia, S. R., Brito, G. M., Seijo, M., Keller, G. A., Somoza, J., et al. (2015). Vitamin D3 seems more appropriate than D2 to sustain adequate levels of 25OHD: a pharmacokinetic approach. Eur. J. Clin. Nutr. 69, 697-702. doi: 10.1038/ejcn.20 15.16

Panarese, A., and Shahini, E. (2020). Letter: Covid-19, and vitamin D. Aliment. Pharmacol. Ther. 51, 993-995. doi: 10.1111/apt.15752

Patrick, R. P., and Ames, B. N. (2015). Vitamin D and the omega-3 fatty acids control serotonin synthesis and action, part 2: relevance for ADHD, bipolar disorder, schizophrenia, and impulsive behavior. FASEB J. 29, 2207-2222. doi: 10.1096/fj.14-268342 
Pereira, M., Dantas Damascena, A., Galvão Azevedo, L. M., de Almeida Oliveira, T., and da Mota Santana, J. (2020). Vitamin D deficiency aggravates COVID19: systematic review and meta-analysis. Crit. Rev. Food Sci. Nutr. 1-9. doi: 10.1080/10408398.2020.1841090

Petrelli, F., Luciani, A., Perego, G., Dognini, G., Colombelli, P. L., and Ghidini, A. (2021). Therapeutic and prognostic role of vitamin D for COVID-19 infection: a systematic review and meta-analysis of 43 observational studies. J. Steroid Biochem. Mol. Biol. 211:105883. doi: 10.1016/j.jsbmb.2021.105883

Robb, C. E., de Jager, C. A., Ahmadi-Abhari, S., Giannakopoulou, P., UdehMomoh, C., McKeand, J., et al. (2020). Associations of social isolation with anxiety and depression during the early COVID-19 pandemic: a survey of older adults in London, UK. Front. Psychiatry 11:591120. doi: 10.3389/fpsyt.2020. 591120

Sabir, M. S., Haussler, M. R., Mallick, S., Kaneko, I., Lucas, D. A., Haussler, C. A., et al. (2018). Optimal vitamin D spurs serotonin: 1,25-dihydroxyvitamin D represses serotonin reuptake transport (SERT) and degradation (MAO-A) gene expression in cultured rat serotonergic neuronal cell lines. Genes Nutr. 13:19. doi: 10.1186/s12263-018-0605-7

Santini, Z. I., Jose, P. E., York Cornwell, E., Koyanagi, A., Nielsen, L., Hinrichsen, C., et al. (2020). Social disconnectedness, perceived isolation, and symptoms of depression and anxiety among older Americans (NSHAP): a longitudinal mediation analysis. Lancet Public Health 5, e62-e70. doi: 10.1016/S2468-2667(19)30 230-0

Smith, B. J., and Lim, M. H. (2020). How the COVID-19 pandemic is focusing attention on loneliness and social isolation. Public Health Res. Pract. 30:3022008. doi: 10.17061/phrp3022008

Song, B. M., Kim, H. C., Rhee, Y., Youm, Y., and Kim, C. O. (2016). Association between serum 25-hydroxyvitamin $\mathrm{D}$ concentrations and depressive symptoms in an older Korean population: a cross-sectional study. J. Affect. Disord. 189, 357-364. doi: 10.1016/j.jad.2015.09.043

Spedding, S. (2014). Vitamin D and depression: a systematic review and metaanalysis comparing studies with and without biological flaws. Nutrients 6, 1501-1518. doi: 10.3390/nu6041501

Teshome, A., Adane, A., Girma, B., and Mekonnen, Z. A. (2021). The impact of vitamin D level on COVID-19 infection: systematic review and meta-analysis. Front. Public Health 9:624559. doi: 10.3389/fpubh.2021.624559

Vellekkatt, F., Menon, V., Rajappa, M., and Sahoo, J. (2020). Effect of adjunctive single dose parenteral vitamin $\mathrm{D}$ supplementation in major depressive disorder with concurrent vitamin D deficiency: a double-blind randomized placebocontrolled trial. J. Psychiatr. Res. 129, 250-256. doi: 10.1016/j.jpsychires.2020. 07.037

Vidgren, M., Virtanen, J. K., Tolmunen, T., Nurmi, T., Tuomainen, T.-P., Voutilainen, S., et al. (2018). Serum concentrations of 25-hydroxyvitamin D and depression in a general middle-aged to elderly population in Finland. J. Nutr. Health Aging 22, 159-164. doi: 10.1007/s12603-017-0948-6

Wacker, M., and Holick, M. F. (2013). Sunlight and vitamin D: a global perspective for health. Dermato Endocrinol. 5, 51-108. doi: 10.4161/derm.24494

Wang, C., Pan, R., Wan, X., Tan, Y., Xu, L., Ho, C. S., et al. (2020). Immediate psychological responses and associated factors during the initial stage of the 2019 coronavirus disease (COVID-19) epidemic among the general population in China. Int. J. Environ. Res. Public Health 17:1729. doi: 10.3390/ ijerph17051729

Xiong, J., Lipsitz, O., Nasri, F., Lui, L. M. W., Gill, H., Phan, L., et al. (2020). Impact of COVID-19 pandemic on mental health in the general population: a systematic review. J. Affect. Disord. 277, 55-64. doi: 10.1016/j.jad.2020.08.001

Yao, Y., Fu, S., Zhang, H., Li, N., Zhu, Q., Zhang, F., et al. (2018). The prevalence of depressive symptoms in Chinese longevous persons and its correlation with vitamin D status. BMC Geriatrics 18:198. doi: 10.1186/s12877-018-0886-0

Zhou, Y.-F., Luo, B.-A., and Qin, L.-L. (2019). The association between vitamin $\mathrm{D}$ deficiency and community-acquired pneumonia: a meta-analysis of observational studies. Medicine (Baltimore) 98:e17252. doi: 10.1097/MD 0000000000017252

Zhu, C., Zhang, Y., Wang, T., Lin, Y., Yu, J., Xia, Q., et al. (2020). Vitamin D supplementation improves anxiety but not depression symptoms in patients with vitamin D deficiency. Brain Behav. 10:e01760. doi: 10.1002/brb3.1760

Conflict of Interest: The authors declare that the research was conducted in the absence of any commercial or financial relationships that could be construed as a potential conflict of interest.

Copyright (c) 2021 Ceolin, Mano, Hames, Antunes, Brietzke, Rieger and Moreira. This is an open-access article distributed under the terms of the Creative Commons Attribution License (CC BY). The use, distribution or reproduction in other forums is permitted, provided the original author(s) and the copyright owner(s) are credited and that the original publication in this journal is cited, in accordance with accepted academic practice. No use, distribution or reproduction is permitted which does not comply with these terms. 